\title{
Impairment of Fear Memory Consolidation in Maternally Stressed Male Mouse Offspring: Evidence for Nongenomic Glucocorticoid Action on the Amygdala
}

\author{
Eun Jeong Lee, ${ }^{\star}$ Gi Hoon Son, ${ }^{\star}$ Sooyoung Chung, Sukwon Lee, Jeongyeon Kim, Sukwoo Choi, \\ and Kyungjin Kim \\ Department of Biological Sciences, Seoul National University, and Brain Research Center for the 21st Century Frontier Program in Neuroscience, Seoul \\ 151-742, Korea
}

\begin{abstract}
The environment in early life elicits profound effects on fetal brain development that can extend into adulthood. However, the longlasting impact of maternal stress on emotional learning remains largely unknown. Here, we focus on amygdala-related learning processes in maternally stressed mice. In these mice, fear memory consolidation and certain related signaling cascades were significantly impaired, though innate fear, fear memory acquisition, and synaptic NMDA receptor expression in the amygdala were unaltered. In accordance with these findings, maintenance of long-term potentiation (LTP) at amygdala synapses, but not its induction, was significantly impaired in the maternally stressed animals. Interestingly, amygdala glucocorticoid receptor expression was reduced in the maternally stressed mice, and administration of glucocorticoids (GCs) immediately after fear conditioning and LTP induction restored memory consolidation and LTP maintenance, respectively, suggesting that a weakening of GC signaling was responsible for the observed impairment. Furthermore, microinfusion of a membrane-impermeable form of GC (BSA-conjugated GC) into the amygdala mimicked the restorative effects of GC, indicating that a nongenomic activity of GC mediates the restorative effect. Together, these findings suggest that prenatal stress induces long-term dysregulation of nongenomic GC action in the amygdala of adult offspring, resulting in the impairment of fear memory consolidation. Since modulation of amygdala activity is known to alter the consolidation of emotionally influenced memories allocated in other brain regions, the nongenomic action of GC on the amygdala shown herein may also participate in the amygdaladependent modulation of memory consolidation.
\end{abstract}

\section{Introduction}

The environment in the womb has long-lasting effects on the later life of offspring, which are known as "programming effects" (Seckl, 2008). For example, the low birth weight of pups from mothers stressed in pregnancy has been correlated with pathophysiological symptoms such as cardiovascular disease and metabolic disorders (Nathanielsz, 1999; Welberg et al., 2001). Moreover, the brain is also vulnerable to environmental challenges in early life that contribute to alterations in the cognition and behaviors of offspring (Welberg et al., 2001; Weinstock, 2008). Previously, we showed that spatial learning associated with hippocampal NMDA receptor-mediated syn-

Received Sept. 7, 2010; revised Feb. 24, 2011; accepted March 24, 2011.

Author contributions: E.J.L., G.H.S., S. Chung, S. Choi, and K.K. designed research; E.J.L., G.H.S., S. Chung, S.L., and J.K. performed research; E.J.L., G.H.S., S. Choi, and K.K. analyzed data; E.J.L., G.H.S., S. Choi, and K.K. wrote the paper. *E.J.L. and G.H.S. contributed equally to this work.

This work was supported by grants from the Korea Ministry of Education, Science, and Technology (MEST) through the Brain Research Center for the 21st Century Frontier R\&D Program in Neuroscience to S. Choi and K.K. E.J.L., S. Chung and J.K. were supported by the Brain Korea 21 Research Fellowships from the MEST.

Correspondence should be addressed to either of the following: Dr. Kyungjin Kim, Department of Biological Sciences, Seoul National University, and Brain Research Center for the $21^{\text {st }}$ Century Frontier Program in Neuroscience, Seoul 151-742, Korea, E-mail: kyungjin@snu.ac.kr; or Dr. Sukwoo Choi, Department of Biological Sciences, Seoul National University, Seoul 151-742, Korea, E-mail: sukw0012@snu.ac.kr.

DOI:10.1523/JNEUROSCI.4692-10.2011

Copyright $\odot 2011$ the authors $\quad 0270-6474 / 11 / 317131-10 \$ 15.00 / 0$ aptic plasticity was defective in maternally stressed mice (Son et al., 2006). However, other prenatal stress-induced defects have not been well characterized in these mice. In the present study, we have questioned whether and how maternal stress affects the amygdala, a major target of stress in the brain that is also known as a key limbic brain region involved in the regulation of emotion, particularly fear-related processes.

The amygdala is an important locus for integrating modulatory influences on emotion through numerous neural and hormonal systems that are affected by stress and that control stress-evoked neurobehavioral alterations (LeDoux, 2007; Joëls and Baram, 2009). Considering such interactions with stress-responsive neuroendocrine systems, it is plausible that the amygdala is a primary target of the programming effects of maternal stress. Indeed, several lines of evidence have suggested that either maternal stress or prenatal glucocorticoid (GC) exposure increases amygdala-dependent anxiety-like behaviors in offspring (Cratty et al., 1995; Welberg et al., 2001; Fan et al., 2009). In this regard, we also have reported that maternally stressed mice were more vulnerable to chronic stress-evoked anxiety-like behaviors associated with hyperactivity of the corticotrophinreleasing hormone (CRH) system in the amygdala (Chung et al., 2005). Despite extensive studies on maternal stress-evoked innate fear and anxiogenic behaviors in offspring, there is as yet little evidence concerning one of the most important amygdala 
functions, i.e., the processing of fear-associated learning and memory.

The basolateral complex of the amygdala (the BLA complex), which is comprised of the lateral, basal, and accessory basal nuclei, is believed to be responsible for the consolidation of emotionally influenced memory (McGaugh, 2000). It is important to note that activation of the stress-responsive hypothalamus-pituitary-adrenal (HPA) neuroendocrine axis and the subsequent secretion of adrenal GC are reported to be heavily involved in aversive memory formation, presumably by exerting an effect on the BLA (Roozendaal et al., 2002; de Quervain et al., 2009; Rodrigues et al., 2009). Therefore, in the present study, we investigated alterations in the GC effects on the BLA in adult male offspring of mothers exposed to prolonged stress during pregnancy. For this purpose, we examined amygdala-based fear memory, maintenance of amygdala LTP, and related signaling events. Last, we examined the possible involvement of nongenomic GC actions on the amygdala of maternally stressed mice.

\section{Materials and Methods}

Animals and the maternal stress procedure. ICR mice, obtained from the Laboratory Animal Center at Seoul National University, were used in all of the experiments and kept in temperature-controlled $\left(22-23^{\circ} \mathrm{C}\right)$ quarters under a $12 \mathrm{~h}$ light/dark photoperiod (lights on at 8:00 A.M.). Standard mouse chow and water were available ad libitum. The maternal stress procedure was performed as described previously (Chung et al., 2005; Son et al., 2006, 2007). Briefly, pregnant ICR mice were prepared by mating with adult males. Pregnant mice of the stressed group were placed individually in a restrainer (a transparent plastic cylinder, $3 \mathrm{~cm}$ in diameter and $9 \mathrm{~cm}$ long) daily for $6 \mathrm{~h}$ (10:00 A.M. to 4:00 P.M.) from $8.5 \mathrm{~d}$ postcoitum ( $\mathrm{dpc}$ ) to 18.5 or $19.5 \mathrm{dpc}$ (the day before parturition). Control pregnant mice remained undisturbed. After weaning on postnatal day 21, the pups born from a stressed mother (STR) were reared in an environment identical to that of the controls (CTL). The STR and CTL groups were separately housed, but three to five mice from different litters were randomly assigned to a cage to exclude possible litter effects. Male offspring at 8-12 weeks of age were used in all experiments. All behavioral tests were performed during the light period (between 11:00 A.M. and 3:00 P.M.). All animal procedures are approved by the Animal Care and Use Committee of Seoul National University.

Passive avoidance test. Adult male mice were trained in an apparatus (PACS-30, Columbus Instruments) in which two compartments were divided by a guillotine door. The larger compartment was made of black Plexiglas and maintained in darkness, while a lamp $(60 \mathrm{~W})$ illuminated the compartment made of white Plexiglas. On the training day, each mouse was placed in the illuminated compartment, facing away from the dark compartment. When the mouse turned around, the door leading to the dark compartment opened. When a mouse entered the dark compartment to the extent that all four paws were on the dark side, the door closed, and a footshock ( $1 \mathrm{~mA}, 3 \mathrm{~s})$ was delivered automatically. Mice were then removed from the apparatus and returned to their home cage. Retention was examined $24 \mathrm{~h}$ later using a procedure similar to that of the training session, except that no footshock was administered. A maximum step-through latency of 3 min was allowed per test session.

Pavlovian fear conditioning. Contextual fear conditioning was performed in the conditioning chamber $(13 \times 13 \times 25 \mathrm{~cm})$ with five repetitions of footshocks $(1 \mathrm{~mA}, 1 \mathrm{~s})$ at $90 \mathrm{~s}$ intervals after habituation for 10 min in the same chamber on the day before training. On the next day, conditioned mice were placed in the same chamber, and the "freezing" time was measured over a period of $5 \mathrm{~min}$. Conditioned freezing was defined as immobility except for respiratory movements. The total freezing time in the test period was represented as a percentage. Auditory fear conditioning was conducted as described previously (Schafe and LeDoux, 2000) with a slight modification. Mice were habituated for $10 \mathrm{~min}$ in the conditioning chamber without disturbance on the day before conditioning. Mice were then placed in the conditioning chamber, and 5 conditioning trial repetitions, each consisting of a tone $(30 \mathrm{~s}, 5 \mathrm{kHz}, 75$
$\mathrm{dB})$ that terminated with a footshock $(1 \mathrm{~mA}, 1 \mathrm{~s})$, were administered. The intertrial interval was $90 \mathrm{~s}$. Conditioned fear responses were tested at the indicated time after conditioning. For each test, mice were placed in a distinct context and reexposed to three tones at 90 s intervals after a $5 \mathrm{~min}$ period of exploration. Freezing behaviors were scored during the tone presentation. The total freezing time in the test period was represented as percentage of the average duration to each tone presentation. Metyrapone (MET; dissolved at $10 \mathrm{mg} / \mathrm{ml}$ in saline; Tocris Bioscience; $100 \mathrm{mg} / \mathrm{kg}$ b.w.; i.p.), a GC synthesis inhibitor or saline as a vehicle, was administered $3 \mathrm{~h}$ before conditioning. Dexamethasone (DEX) 21-phosphate disodium salt (dissolved at $100 \mu \mathrm{g} / \mathrm{ml} \mathrm{3 \%}$ ethanol in saline; Sigma; $1 \mathrm{mg} / \mathrm{kg}$ b.w.; s.c.), corticosterone (CORT; dissolved at $50 \mu \mathrm{g} / \mathrm{ml}$ in $3 \%$ ethanol in saline; Sigma; $0.5 \mathrm{mg} / \mathrm{kg}$ b.w.; s.c.), or vehicle ( $3 \%$ ethanol in saline) was injected immediately after training.

Measurement of spontaneous motor activity. Spontaneous motor activity in the fear conditioning chamber was measured under conditions similar to those for fear memory testing. Mice were habituated in the tone-testing chamber $(13 \times 13 \times 25 \mathrm{~cm})$ with a bedded floor for $10 \mathrm{~min}$ on the day before measuring the activities. On the next day, mice were placed in the same chamber, and then spontaneous motor activities were recorded for $10 \mathrm{~min}$ (the time period required for the tone testing) and analyzed using the EthoVision Pro system (Noldus Information Technology). The distance moved was used as an index of motor activity.

Unconditioned fear response to predator odor. To examine innate fear, mice were placed in the chamber with a beaker containing $30 \mu \mathrm{l}$ of a synthetic fox feces odor, trimethylthiazoline (TMT; PheroTech), which was reported previously to elicit freezing in rodents (Wallace and Rosen, 2001). TMT-evoked freezing was recorded for $10 \mathrm{~min}$ after TMT exposure and scored as a percentage of the time spent in the freezing behavior at $2 \mathrm{~min}$ intervals. Freezing behavior was defined the same as in the Pavlovian fear conditioning case.

Tissue preparation and immunoblot analysis. For tissue preparation, mice were killed between 11:00 A.M. and 2:00 P.M., and the brain was quickly removed and placed on ice. The amygdala, hippocampus, and hypothalamus were dissected from 1-mm-thick brain slices prepared with a brain matrix and quick frozen in liquid nitrogen. Whole-cell lysates and postsynaptic density proteins were prepared as previously described (Son et al., 2006). Protein samples were resolved by SDS-polyacrylamide gel and transferred to polyvinylidene difluoride (PVDF) membranes (Millipore) in a Bio-Rad Trans-Blot electrophoresis apparatus with Towbin's buffer (25 mu Tris, pH 8.3, $192 \mathrm{~mm}$ glycine, and 20\% methanol). The blots were blocked in Tris-buffered saline (TBS; $150 \mathrm{~mm} \mathrm{NaCl}, 10 \mathrm{~mm}$ Tris, pH 7.6, and $2 \mathrm{mM} \mathrm{MgCl}_{2}$ ) containing $0.5 \%$ Tween 20 and $5 \%$ bovine serum albumin (BSA), and incubated with primary antibodies at room temperature for $1 \mathrm{~h}$. The blots were then washed four times with TBS/0.5\% Tween 20 . The bound primary antibody was subsequently detected by incubation with secondary antibodies linked to horseradish peroxidase (Jackson ImmunoResearch). The blots were then washed four more times. Immunoreactive bands were visualized with GE Healthcare ECL reagents according to the manufacturer's instructions (GE Healthcare). The blots were analyzed with Bio1D image analysis software (Vilber-Lourmat) and were expressed as relative optical densities (R.O.D.). The antibodies used in the immunoblotting were as follows: anti-GluN1 (BD Bioscience); GluN2A, GluN2B, and GluA1 (Millipore); actin (Sigma); PSD-95, extracellular-regulated kinase 1 and 2 (ERK1/2), phosphorylated ERK1/2, cAMP response element binding protein (CREB), and phosphorylated CREB (Cell Signaling Technology); and glucocorticoid receptor (GR) and mineralocorticoid receptor (MR) (Santa Cruz Biotechnology). These antibodies are all commercially available.

Immunohistochemistry. Mice were killed $15 \mathrm{~min}$ after fear conditioning and DEX treatment. Brains were removed and cut into 3-mm-thick sections with a brain matrix, including the amygdala. The sectioned brain tissues were postfixed in PBS, pH 7.6, containing 4\% paraformaldehyde overnight at $4^{\circ} \mathrm{C}$ and then cryoprotected in $30 \%$ sucrose for $24 \mathrm{~h}$ at $4^{\circ} \mathrm{C}$. Frozen coronal sections were cut at a $20 \mu \mathrm{m}$ thickness on a cryostat. After blocking with PBS containing 10\% normal horse serum and $0.3 \%$ Triton $\mathrm{X}-100$, slices were incubated at room temperature for $1 \mathrm{~h}$ in antiphosphorylated ERK1/2 (at 1:200 dilutions; Cell Signaling Technology) in PBS-3\% horse serum- $0.1 \%$ Triton X-100. After several washes with 
PBS, Cy3-conjugated anti-rabbit IgG antibody (1:500) was applied for 30 min. Subsequently, sections were washed, mounted, and observed under fluorescence microscopy (Axiovert 200M, Carl Zeiss). Sections from comparable anteroposterior levels (1.3-1.9 $\mathrm{mm}$ posterior to the bregma) containing the BLA regions were selected for quantifying nuclear pERK1/ 2-positive cells. Cell counts were taken from at least three sections per mouse and scored using a defined boundary approximately equivalent to the size of the BLA.

Determination of serum GC levels. Mice were killed between 1:00 P.M. and 2:00 P.M. Trunk blood was collected and centrifuged at 10,000 $\times g$ for $20 \mathrm{~min}$ to obtain serum. Serum samples were quick frozen and stored at $-70^{\circ} \mathrm{C}$ until use. GC levels were assayed using a commercial radioimmunoassay kit, according to the manufacturer's instructions (Diagnostic Products).

Electrophysiology. Electrophysiological experiments were performed as described in a previous report (Kim et al., 2007b). Control mice and maternally stressed mice were anesthetized with halothane and killed. The isolated brains were placed in an ice-cold modified artificial CSF (aCSF) solution containing the following: $175 \mathrm{~mm}$ sucrose, $20 \mathrm{~mm} \mathrm{NaCl}$, $3.5 \mathrm{~mm} \mathrm{KCl}, 1.25 \mathrm{~mm} \mathrm{NaH}_{2} \mathrm{PO}_{4}, 26 \mathrm{~mm} \mathrm{NaHCO}_{3}, 1.3 \mathrm{~mm} \mathrm{MgCl}_{2}$, and 11 mM D-(+)-glucose, and aerated with $95 \% \mathrm{O}_{2} / 5 \% \mathrm{CO}_{2}$. Coronal slices (300 $\mu \mathrm{m}$ for whole-cell recordings and $400 \mu \mathrm{m}$ for field recordings), including the lateral nucleus in the amygdala (LA), were cut and incubated in normal aCSF containing the following: $120 \mathrm{~mm} \mathrm{NaCl}, 3.5 \mathrm{~mm}$ $\mathrm{KCl}, 1.25 \mathrm{~mm} \mathrm{NaH}_{2} \mathrm{PO}_{4}, 26 \mathrm{~mm} \mathrm{NaHCO}, 1.3 \mathrm{~mm} \mathrm{MgCl} 2,2 \mathrm{~mm} \mathrm{CaCl}$, and $11 \mathrm{~mm} \mathrm{D}-(+)$-glucose, then continuously bubbled at room temperature with $95 \% \mathrm{O}_{2} / 5 \% \mathrm{CO}_{2}$. A submersion-type recording chamber was continuously superfused with aCSF $\left(31.0-33.0^{\circ} \mathrm{C}\right.$ for whole-cell recordings and $33.0-34.5^{\circ} \mathrm{C}$ for field recordings) containing picrotoxin (100 $\mu \mathrm{M}$ for whole-cell recordings and $10 \mu \mathrm{M}$ for field-potential recordings). We included picrotoxin in the recording solution to isolate excitatory synaptic transmission and to block feedforward GABAergic inputs to principal neurons in the LA. For field potential recordings, where afferents were stimulated at a stronger intensity, a lower concentration of picrotoxin was used to preserve the inhibitory tone and thus prevent multisynaptic firing. We chose brain slices containing a well isolated, sharply defined trunk (containing thalamic afferents) crossing the dorsolateral division of the LA, which is a site of convergence for somatosensory and auditory inputs (Pitkänen et al., 1997). The sizes of the LA and central amygdala were relatively constant in these slices, and the trunk closest to the central nucleus of the amygdala was used when multiple trunks were observed. Thalamic afferents were stimulated using a concentric bipolar electrode (MCE-100, Rhodes Medical Instruments) placed on the midpoint of the trunk between the internal capsule and medial boundary of the LA. Regions and cells of interest for all recordings were located beneath the midpoint of the trunk horizontally spanning the LA. Whole-cell currents were filtered at $1 \mathrm{kHz}$, then digitized at 20 $\mathrm{kHz}$ using a MultiClamp 700A digitizer (Molecular Devices). Recordings were obtained by using pipettes with series resistances of 2.5-3.5 $\mathrm{M} \Omega$ when filled with the following solution: $100 \mathrm{~mm}$ Cs-gluconate, $0.6 \mathrm{~mm}$ EGTA, 10 mm HEPES, $5 \mathrm{~mm} \mathrm{NaCl}, 20 \mathrm{~mm}$ tetraethylammonium, $4 \mathrm{~mm}$ Mg-ATP, 0.3 mm Na-GTP, and 3 mm QX314, with the $\mathrm{pH}$ adjusted to 7.2. While voltage clamped, a minor proportion $(<5 \%)$ of recorded neurons exhibited spontaneous EPSCs with faster decay times and larger amplitude $(>100 \mathrm{pA})$. As these are characteristic of inhibitory neurons, they were excluded from the analysis (Kim et al., 2007b). Extracellular field potentials were recorded by using a parylene-insulated microelectrode (573210; A-M Systems) in 400- $\mu \mathrm{m}$-thick slices. As shown in previous studies (Huang et al., 2000; Kim et al., 2007b, 2009), field potentials (population spikes) at thalamo-LA synapses exhibited a constant and short latency of $\sim 4 \mathrm{~ms}$, reliably followed by high-frequency stimulation without failure, and could be blocked by kynurenic acid. This indicates that the field potentials measured in the present study reflect glutamatergic and monosynaptic responses at thalamo-LA synapses. LTP was induced by six trains of high-frequency stimuli (HFS; $100 \mathrm{~Hz}, 1 \mathrm{~s}$ duration, $1 \mathrm{~min}$ interval) with the same intensity and pulse duration as the baseline stimuli. Vehicle, DEX (dissolved in aCSF at a final concentration of 20 $\mathrm{nM}$ ), or BSA-conjugated DEX (DEX-BSA; dissolved in aCSF at a final concentration of $200 \mathrm{nM}$ ) was applied immediately after six repetitions of
HFS for $30 \mathrm{~min}$. To obtain stable, long-term recordings, we began field recordings at least $3 \mathrm{~h}$ after preparation of the slices. To improve the signal-to-noise ratio, data were averaged using a three-point running average in time-lapse experiments.

Cannula implantation and infusion of DEX-BSA. Cannula implantation was performed as described previously (Kim et al., 2007a). Mice were anesthetized with sodium pentobarbital (50 mg/kg, i.p.), mounted on a stereotaxic apparatus (David Kopf Instruments), and bilaterally implanted with 26 gauge stainless steel cannulas (model C315G; Plastic Products) into the BLA complex (AP $-1.3 \mathrm{~mm}, \mathrm{ML} \pm 3.6 \mathrm{~mm}, \mathrm{DV}-3.8 \mathrm{~mm}$ ). A 32 gauge dummy cannula was inserted into each guide cannula to prevent clogging. Two jewelry screws were implanted over the skull to serve as anchors, and the whole assembly was affixed on the skull with dental cement. Mice were given at least 1 week to recover before experimentation. After completion of the experiments, correct placement of the injection cannula tips was verified in all animals. For this purpose, brains were removed from animals and fixed overnight in $\mathrm{PBS} / 4 \%$ paraformaldehyde. The fixed brains were coronally sectioned into $70 \mu \mathrm{m}$ thicknesses using a vibratome (World Precision Instruments). Sections were then stained with cresyl violet and examined under light microscopy. DEX-BSA dissolved in saline ,tk; $2(2 \mu \mathrm{g} / \mu \mathrm{l})$ was administrated bilaterally into the BLA complex via a 33 gauge injector cannula (C315I; Plastic Products) attached to a $10 \mu \mathrm{l}$ Hamilton syringe. A solution containing $0.4 \mu \mathrm{g}$ of DEX-BSA in $0.2 \mu \mathrm{l}$ was administered immediately after auditory fear conditioning at a rate of $0.2 \mu \mathrm{l} / \mathrm{min}$. After infusion, injector cannulas were left in place for an additional minute to allow the solution to diffuse away from the tip.

Data analysis. The results from behavioral studies, quantitative immunoblot analyses, and serum GC levels were statistically evaluated using Student's $t$ test and/or ANOVA followed by Newman-Keuls test as a post hoc comparison. Two-way repeated-measure (RM)-ANOVA followed by Student's $t$ test for each time point or one-way ANOVA was used for statistical evaluation of the average of population spike amplitudes. A probability level of $p<0.05$ was chosen to denote statistical significance.

\section{Results}

Impaired contextual fear memory in maternally stressed mice To determine whether chronic maternal stress has a long-lasting effect on emotional learning processes, male adult offspring were subjected to a passive avoidance test and contextual fear conditioning, which are well established behavioral paradigms for investigating fear-related memory associated with contextual information.

In the passive avoidance test, both the control and maternally stressed mice displayed similarly short step-through latencies of $<20$ s before receiving a footshock (training). Control mice exhibited significantly increased latency to enter into the dark compartment where they had previously received a footshock in the test sessions $24 \mathrm{~h}$ after training. However, the maternally stressed mice entered the dark compartment with much shorter latency than controls $\left(F_{(1,56)}=30.31, p<0.01\right.$ for group differences; $F_{(1,56)}=39.22, p<0.01$ for training; $F_{(1,56)}=28.07, p<0.01$ for interaction) (Fig. 1A). Impaired fear-associated learning in maternally stressed mice was also revealed by a contextual fear conditioning paradigm. Control and maternally stressed mice freely explored a fear conditioning chamber without any significant difference in freezing behaviors before they were exposed to footshock (training) in the course of contextual fear conditioning (Fig. $1 B$ ). However, on the next day after conditioning, the maternally stressed mice displayed fewer freezing behaviors in the same conditioning chamber than the control mice $\left(F_{(1,36)}=\right.$ 20.36, $p<0.01$ for group differences; $F_{(1,36)}=208.2, p<0.01$ for training; $F_{(1,36)}=21.8, p<0.01$ for interaction) (Fig. $1 B$ ). Together, these results show that maternally stressed adult mice have a defect in fear memory that is related to contextual information. Since maternal stress affects NMDA receptor-mediated synaptic functions in the hippocampus (Son et al., 2006), it is 

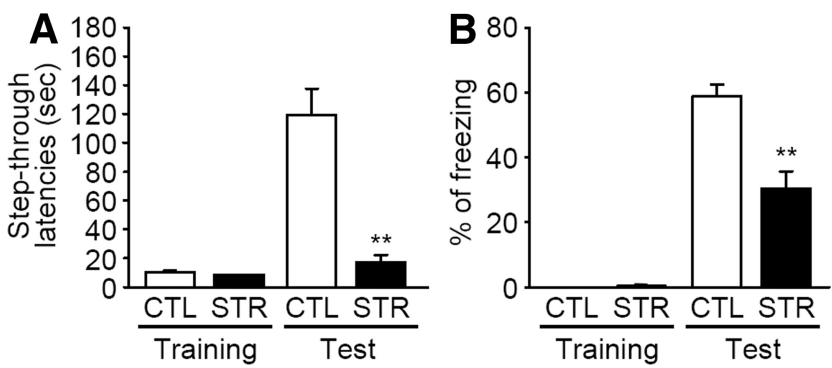

Figure 1. Fear memory impairment in maternally stressed mice in a passive avoidance test and contextual fear conditioning. $\boldsymbol{A}$, Step-through latencies into the dark compartment before (training) and after a footshock (test) are expressed as the means \pm SEM $\left({ }^{* *} p<0.01\right.$ vs (TL test by Newman-Keuls test; $n=15$ for each group). $B$, Freezing percentage before (training) and after pairing of the context and footshock (test) ${ }^{* *} p<$ 0.01 vs (TL test by Newman-Keuls test; $n=10$ for (TL and $n=11$ for STR).

likely that the memory impairments shown in the two tasks are due, at least in part, to a malfunctioning of the hippocampus, which is critical for the acquisition of contextual information.

\section{Impaired auditory fear memory in maternally stressed mice}

Unlike contextual fear conditioning, auditory-cued Pavlovian fear conditioning is known to be dependent on the amygdala, not the hippocampus (Fanselow and LeDoux, 1999). To test the possibility that amygdala functions are altered in maternally stressed mice, we examined whether these mice normally acquire conditioned fear responses after auditory-cued fear conditioning. In the auditory fear conditioning paradigm in rodents, the convergence of a neutral-conditioned stimulus (CS) and an aversive unconditioned stimulus (US) leads to fear responses, such as freezing behavior. We tested the fear memory in the animals by measuring the freezing time in two different phases: the acquisition, i.e., initial learning of the association of CS with US during the training of fear conditioning, and the retrieval of conditioned fear, a test of the CS-US association in which the CS is presented alone. In the acquisition session, an auditory tone serving as the CS was paired with a footshock in five successive trains. The freezing behavior of both the control and maternally stressed mice increased over the number of training sessions without any significant difference in the acquisition phase, indicating that maternal stress does not significantly alter auditory fear memory acquisition in adult offspring $\left(F_{(1,56)}=0.054, p=0.82\right.$ for group differences by RM-ANOVA) (Fig. $2 A$ ). However, freezing was found to be much weaker in the maternally stressed mice compared with control mice when tested $24 \mathrm{~h}$ after conditioning (controls, $53.36 \pm 9.26$ vs maternally stressed mice, $12.87 \pm$ $7.31 \%$ of freezing; $p<0.01 ; n=8$ for each group) (Fig. $2 B$ ), indicating that the acquired fear memory may not be effectively maintained in maternally stressed mice.

Although both groups showed similar freezing responses during the acquisition phase, it cannot be ruled out that the impairment in fear memory retention originated from a decrease in freezing behavior due to unknown reasons (e.g., differences in the expression of fear and/or motor behavior), rather than the fear-associated learning process. We first hypothesized that changes in the unlearned fear response may alter freezing in maternally stressed mice. To test this, we compared innate fear elicited by a synthetic fox feces odor (TMT), which is known to elicit freezing behaviors in rodents as a predator odor stimulus (Wallace and Rosen, 2001), between the maternally stressed and control mice. Mice in the two groups exhibited similar freezing responses over time when exposed to $\operatorname{TMT}\left(F_{(1,40)}=0.039, p=\right.$
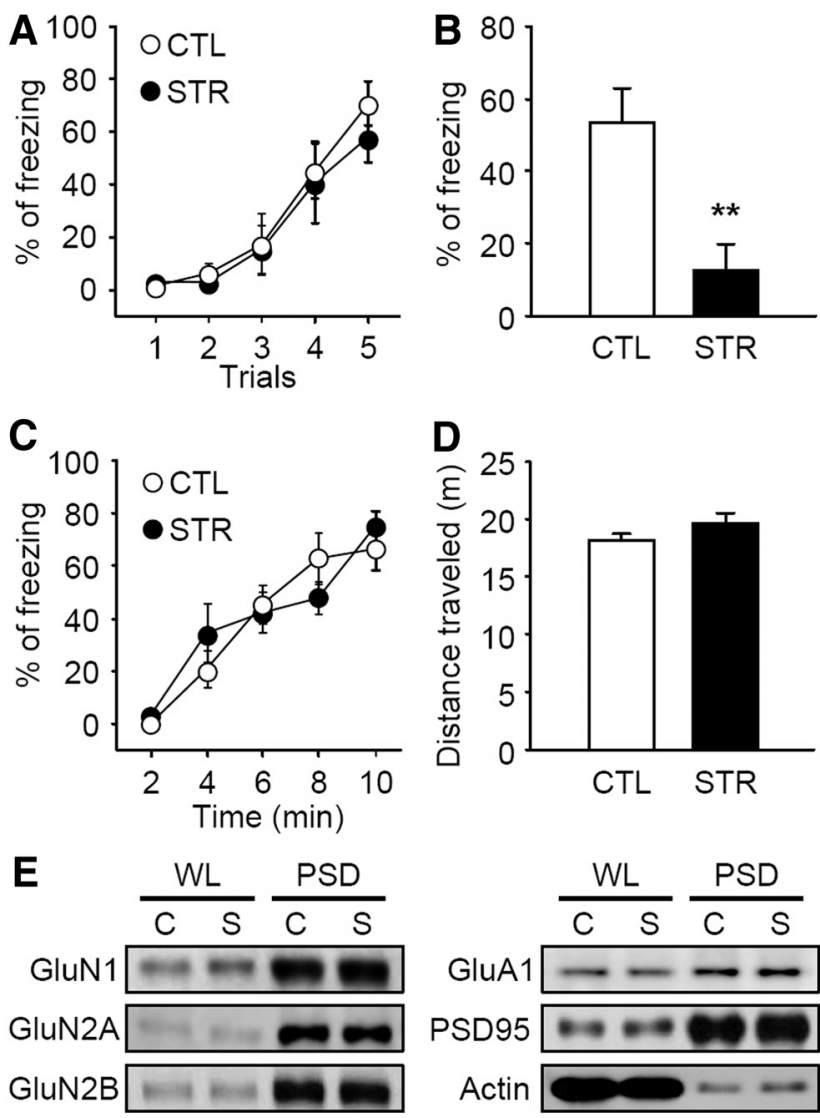

Figure 2. Impaired retrieval of auditory fear memory, but not fear memory acquisition, in maternally stressed mice. $\boldsymbol{A}$, Fear memory acquisition curves represented as the percentage of freezing behavior during five repetitions of tone and footshock pairing. $\boldsymbol{B}$, The freezing percentage during tone exposure without footshock at $24 \mathrm{~h}$ after training is presented as the means \pm SEM $\left({ }^{* *} p<0.01\right.$ vs CTL by Student's $t$ test; $n=8$ for each group). C, Freezing behaviors in response to a predator odor during a $10 \mathrm{~min}$ trial. Freezing was measured by at 2 min intervals ( $n=6$ for each group). $\boldsymbol{D}$, Spontaneous motor activities for $10 \mathrm{~min}$ in the test chamber are expressed as the means \pm SEM of the distance traveled ( $p=0.185$ between two groups by Student's $t$ test; $n=6$ for each group). $\boldsymbol{E}$, Immunoblot analysis of synaptic molecules in whole-cell lysates (WL) and PSD-enriched fractions (PSD) from dissected amygdala of the control and maternally stressed mice. C and $S$ represent control and maternally stressed groups, respectively.

0.847 for group differences by RM-ANOVA) (Fig. 2C), showing that not only unlearned fear, but also fear expression (freezing), was unaltered in maternally stressed mice. Then we tested the possibility that hyperlocomotion alters freezing in maternally stressed mice, since the maternally stressed mice exhibited impaired habituation to a novelty with hyperlocomotion (Son et al., 2007). After 10 min habituation (see Materials and Methods for additional details), the two groups of mice were placed in the tone-testing chamber for $10 \mathrm{~min}$ (the time period required for the tone testing) to estimate spontaneous locomotive activity. However, we failed to find any significant difference in spontaneous motor activity between the two groups of mice under similar conditions of auditory fear testing (controls, $18.13 \pm 0.59$ vs maternally stressed mice, $24.59 \pm 0.89 \mathrm{~m}$ of the distance traveled; $p=0.19 ; n=6$ for each group) (Fig. $2 D$ ), ruling out the possibility that freezing changes are due to hyperlocomotive activity in maternally stressed mice. Together, these findings suggest that freezing is a reliable measure of learned fear in maternally stressed mice, and that learned fear, but not unlearned fear, is specifically impaired in these mice. 
Considering the essential roles of the glutamate receptors in the amygdala in the acquisition of fear memory (Goosens and Maren, 2004; Rodrigues et al., 2004), we examined the synaptic association of the glutamate receptor subunits GluN1 and GluN2A/B (subunits of NMDA receptor) as well as GluA1 (a subunit of AMPA receptor) in the amygdala extracts. There were no obvious differences between the two groups in terms of protein expression levels or synaptic association of these molecules, as revealed by immunoblot analyses of both the whole-cell lysate and postsynaptic density (PSD) protein-enriched fraction prepared from the amygdala (Fig. 2E). These findings are in contrast to our previous study, in which synaptic protein composition in the hippocampus was found to be altered in maternally stressed mice (Son et al., 2006).

\section{Effect of maternal stress on consolidation of auditory fear memory}

Memory consolidation is a process of converting short-term memory (STM) into stable long-term memory (LTM) over a period of time after the acquisition of information (McGaugh, 2000). Auditory fear memory encoded in the amygdala has been shown to be consolidated within $24 \mathrm{~h}$ and short-term fear memory is known to be maintained for up to $4 \mathrm{~h}$ (Schafe and LeDoux, 2000). Therefore, if there is a defect in the consolidation of auditory fear memory, memory retention assessed $24 \mathrm{~h}$ after conditioning would be impaired, while memory retention assessed within $4 \mathrm{~h}$ after conditioning would be intact. To determine whether the consolidation of auditory fear memory is defective in maternally stressed mice, we compared freezing behaviors in response to CS between maternally stressed and control mice at different time points after auditory fear conditioning. Maternally stressed mice exhibited reduced freezing at time points $>4 \mathrm{~h}$ after conditioning compared with control mice, while they exhibited unaltered freezing at time points within $2 \mathrm{~h}$ after conditioning $\left(F_{(1,76)}=21.04, p<0.01\right.$ for group differences; $F_{(4,76)}=15.81, p<0.01$ for time differences, and $F_{(4,76)}=4.593, p<0.01$ for interaction; $p<0.01$ between control and maternally stressed mice at 4 and $24 \mathrm{~h}$ after auditory fear conditioning) (Fig. $3 A$ ). These findings suggest that prenatal stress impairs the consolidation of auditory fear memory in adult offspring.

Consolidation of auditory fear memory requires the activation of MAPKs (mitogen-activated protein kinases), particularly ERK1/2, and active ERK1/2 stimulates a subset of transcription factors, including CREB proteins in the amygdala, which in turn promotes the gene expression required for maintaining longterm fear memory (Schafe et al., 2000; Stanciu et al., 2001; Rodrigues et al., 2004). To determine whether the activation of ERK1/2 and CREB is also impaired in maternally stressed mice, phosphorylation of ERK1/2 and CREB in the amygdala was examined by immunoblotting at 15, 60, and 240 min after auditory fear conditioning (Fig. 3B). Control mice exhibited rapid and transient increases in phosphorylation of both ERK1/2 and CREB after fear conditioning, whereas maternally stressed mice exhibited impairment in the conditioning-induced phosphorylation of both of them. Thus, these findings strengthen the notion that fear memory consolidation processes are impaired in maternally stressed mice.

Effects of GC on auditory fear memory consolidation and ERK1/2 phosphorylation in maternally stressed mice Adrenal GC hormone is known to be involved in the consolidation of aversive memory (Rodrigues et al., 2009). Importantly, we
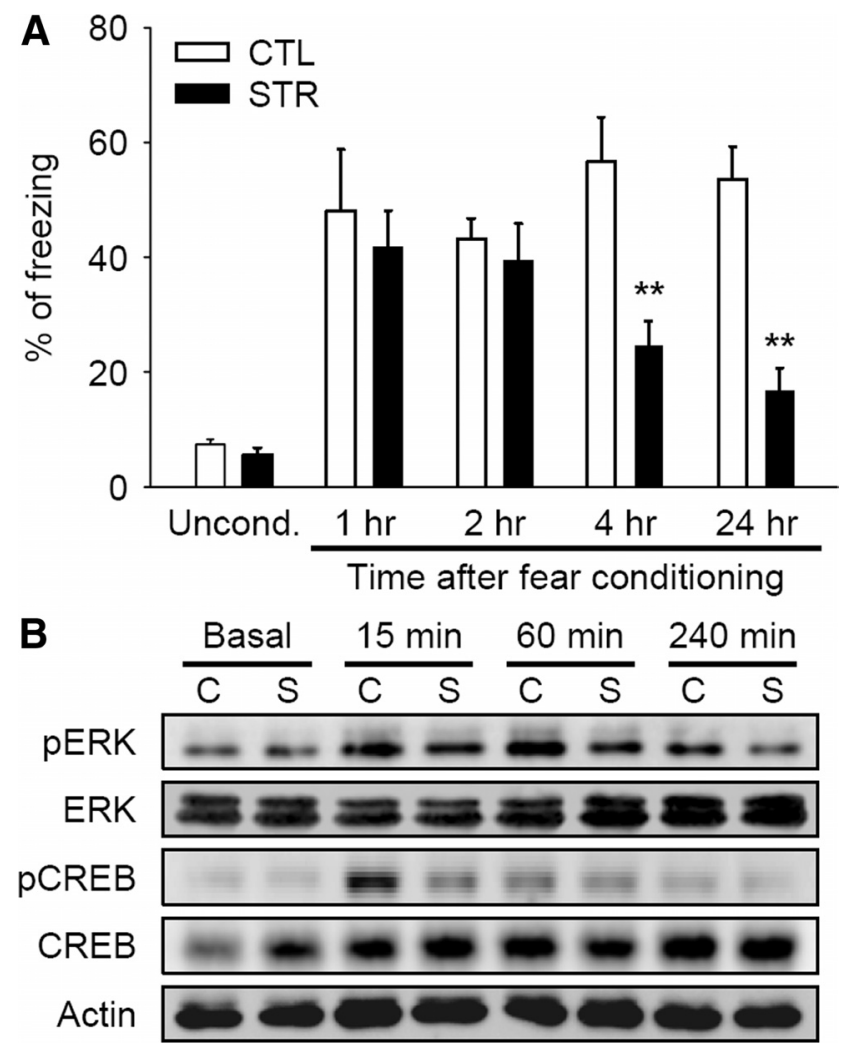

Figure 3. Impairment of auditory fear memory consolidation. $\boldsymbol{A}$, Fear responses to the auditory cue in the unconditioned mice (Uncond.) or conditioned mice at 1, 2, 4, or $24 \mathrm{~h}$ after training $\left({ }^{* *} p<0.01\right.$ vs CTL at the same time after conditioning by Newman-Keuls test; $n=$ 6-12 for each group). $\boldsymbol{B}$, Immunoblot analyses of ERK $1 / 2$ and (REB phosphorylation on the amygdala whole-cell lysates prepared at 15, 60, or 240 min after fear conditioning. Each sample was prepared by pooling 3 -4 animals. Actin was used as an internal control to ensure a similar loading amount.

have reported that prenatal stress alters the HPA neuroendocrine axis reactivity, which controls adrenal GC secretion in adult offspring (Chung et al., 2005). It is therefore conceivable that alterations in the GC signaling cascade may account for the observed defects in fear memory consolidation in maternally stressed mice. To test this, we examined the effects of MET (a GC synthesis inhibitor), CORT (an endogenous rodent GC), and DEX (a synthetic GC) on fear memory retention assessed $24 \mathrm{~h}$ after auditory fear conditioning (Fig. 4A). MET-injected control mice (a systemic injection $3 \mathrm{~h}$ before fear conditioning) exhibited reduced freezing compared with vehicle-injected control mice. In contrast, CORT-injected maternally stressed mice (a systemic injection immediately after conditioning) exhibited increased freezing comparable to that observed in control mice injected with either vehicle or CORT. DEX (a systemic injection immediately after conditioning), which has a higher affinity for the GR than MR, mimicked the effect of CORT (for controls, $F_{(3,23)}=134.3, p<$ 0.01 ; for maternally stressed mice, $F_{(3,27)}=248.4, p<0.01$ by one-way ANOVA). Thus, these findings suggest that pharmacological activation of GRs immediately after fear conditioning restores fear memory consolidation in maternally stressed mice.

Since pharmacological activation of GRs apparently restores fear memory consolidation in maternally stressed mice, we reasoned that DEX injection after conditioning also restores conditioning-induced phosphorylation of ERK1/2 in these mice. To test this, we examined expression levels of phosphorylated ERK1/2 in the whole-cell extracts of the amygdala by immunoblotting at $15 \mathrm{~min}$ after auditory 
fear conditioning (for controls, $F_{(3,14)}=$ 18.86, $p<0.01$; for maternally stressed mice, $F_{(3,14)}=36.51, p<0.01$ by one-way ANOVA) (Fig. 4B,C). Prenatal stress impaired the conditioning-induced phosphorylation of ERK1/2 in adult offspring, whereas DEX injection after conditioning apparently restored it in these mice. In contrast to the postconditioning administration, DEX injection without conditioning did not significantly affect ERK1/2 phosphorylation levels in either group, indicating that the enhancing effect of DEX on ERK1/2 phosphorylation requires fear conditioning.

To more precisely examine whether restoration of ERK1/2 phosphorylation in stressed animals takes place in the BLA complex, we compared the number of phosphorylated ERK1/2-immunoreactive (pERK-ir) cells in this region by the same condition used in the immunoblot analyses (for controls, $F_{(3,20)}=4.754, p<0.05$; for maternally stressed mice, $F_{(3,20)}=$ $12.05, p<0.01$ by one-way ANOVA) (Fig. $4 D, E)$. As in the results obtained by immunoblotting, fear conditioning significantly increased the number of pERK-ir cells in control mice, but not in maternally stressed mice. Also, in maternally stressed mice, postconditioning injection of DEX increased the number of pERK-ir cells to levels comparable with those in the fearconditioned control mice.

Collectively, our findings show that exogenously injected GC restores both ERK1/2 phosphorylation and fear memory consolidation in maternally stressed mice. The restored phosphorylation of ERK1/2 after conditioning appears to mediate the restorative effect of GC on fear memory consolidation.

\section{Serum GC profiles after auditory fear conditioning and the basal GC receptor level}

In the next set of experiments, we intended to further address the molecular and cellular changes underlying the impaired memory consolidation observed in maternally stressed mice. We first examined changes in serum GC profiles after auditory fear conditioning. Both control and maternally stressed mice exhibited a significant elevation in serum GC levels $30 \mathrm{~min}$ after conditioning. The increased GC levels returned to baseline in the control mice within $180 \mathrm{~min}$, whereas the increased GC levels in the maternally stressed mice were maintained at significantly higher levels than in the control mice $\left(F_{(1,22)}=4.390, p<0.05\right.$ for group differences; $F_{(2,22)}=32.39$, $p<0.01$ for time differences; $F_{(2,22)}=8.816, p<0.05$ for interaction) (Fig. 5A). Thus, fear conditioning appears to evoke a prolonged incremental increase in the circulating GC levels in maternally stressed mice. This may result from impaired GC signaling resulting in a reduction in the feedback inhibition of GC secretion.
The actions of circulating GC are mediated by binding either to the high-affinity MR or to the low-affinity GR (De Kloet, 2004). The results shown in Figures 4 and $5 A$ demonstrate that maternally stressed mice are much less sensitive to elevated serum GC after auditory fear conditioning than control mice. One possible explanation is that a lower receptor expression level accounts for the lower sensitivity to GC in maternally stressed mice. To test this idea, we examined the GR and MR expression levels in the amygdala, hippocampus, and hypothalamus, all of which are major target sites of GC in the brain (Fig. $5 B$ ). The GR protein expression was significantly reduced in all tested tissues in the maternally stressed mice compared with the control mice (amygdala: $58.73 \pm 6.86 \%$ of controls in normalized R.O.D., $p<$ 0.01; hippocampus: $39.81 \pm 7.28 \%, p<0.01$; hypothalamus: $63.11 \pm 8.74 \%, p<0.05 ; n=6$ for each group). In contrast to $\mathrm{GR}$, the basal expression of MR was not significantly altered in 

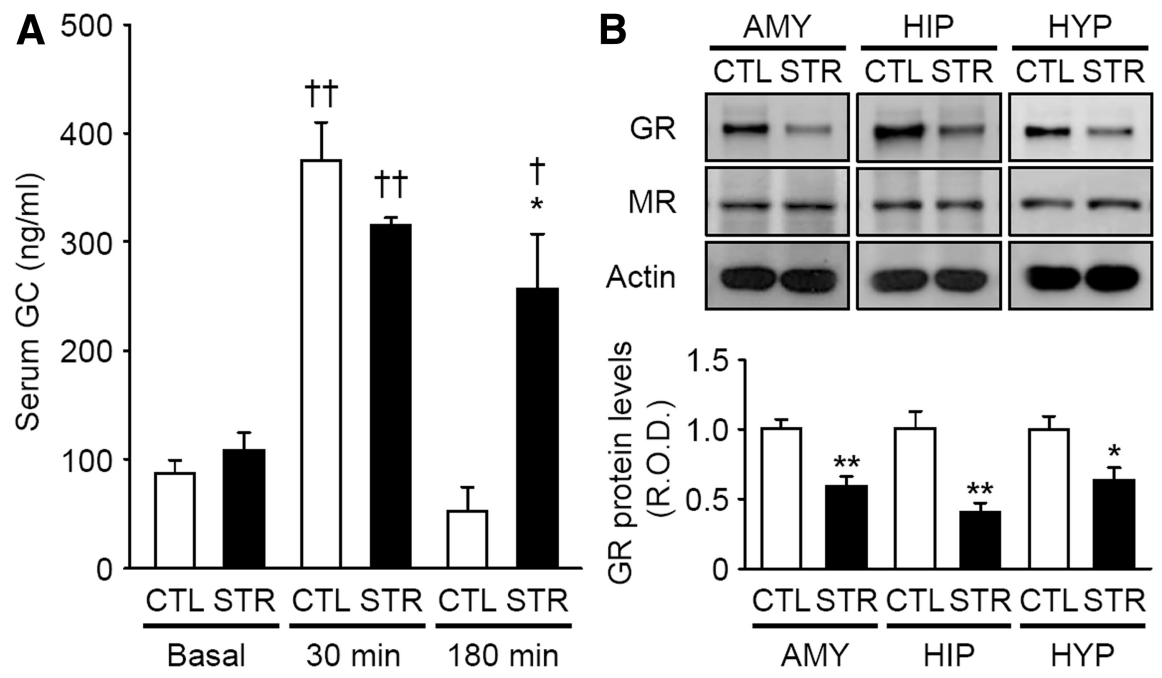

Figure 5. Serum $\mathrm{GC}$ levels in response to auditory fear conditioning and expression of $\mathrm{GC}$ receptors in various brain regions. $A$, Radioimmunoassay of serum $\mathrm{GC}$ levels from sacrificed mice without training (basal) or 30 and 180 min after fear conditioning $\left({ }^{*} p<0.05\right.$ vs CTL at the same time point; ${ }^{\dagger} p<0.05$ and ${ }^{+\dagger} p<0.01$ vs basal serum $\mathrm{GC}$ levels by Newman-Keuls post hoc comparison; $n=4-6$ for each group). $B$, Protein levels of the GR and MR in the amygdala (AMY), hippocampus (HIP), and hypothalamus (HYP), with actin as an internal control. Relative GR levels are presented in the lower panel as the means \pm SEM of R.O.D., where the mean value from each CTL tissue is set as $1\left({ }^{*} p<0.05\right.$ and ${ }^{* *} p<0.01$ vs (TL by Student's $t$ test; $\left.n=6\right)$.

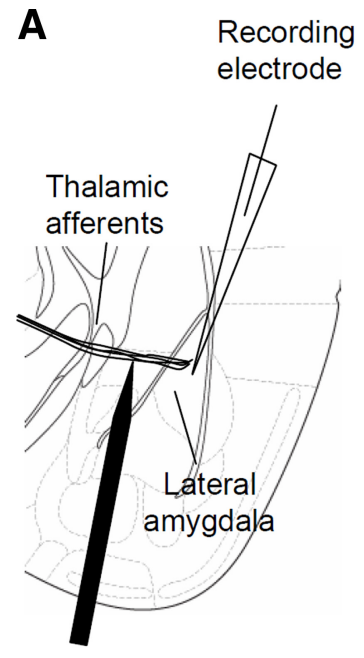

Stimulus electrode
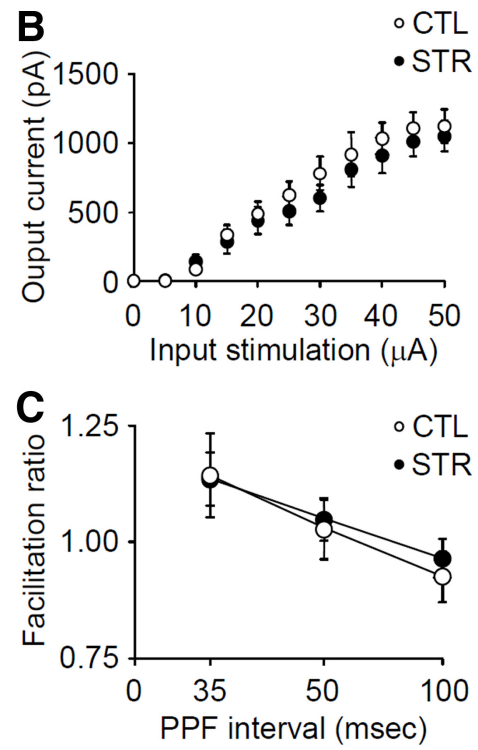

Figure 6. Basal synaptic transmission in the thalamo-LA pathway of mouse brain slices. $\boldsymbol{A}$, Schematic representation of a brain slice containing the amygdala showing the position of the recording and stimulation pipettes. $\boldsymbol{B}$, Input- output curves for evoked EPSCs in the CTL and STR groups ( $n=7$ for each group). C, Paired-pulse facilitation in amygdaloid neurons with 35, 50, or 100 ms interpulse intervals ( $n=8$ for CTL and $n=11$ for STR).

any of the tissues examined. Therefore, it is suggested that the reduced GR expression is responsible for the impairment in memory consolidation and conditioning-evoked ERK1/2 phosphorylation in the amygdala of maternally stressed mice.

\section{Basal synaptic transmission in the thalamo-LA pathway}

It is reasonable that altered basal transmission in the thalamo-LA pathway might be responsible for the observed impairment in maternally stressed mice, since this pathway is one of the major excitatory inputs to the BLA complex, particularly during auditory-cued fear conditioning (Pitkänen et al., 1997; LeDoux, 2007). To test this possibility, we examined excitatory synaptic transmission in the thalamo-LA pathway by measuring both the input-output relation and paired-pulse facilitation (PPF) ratio of EPSCs using a whole-cell patch-clamp method (Fig. 6A). EPSCs recorded with different stimulus intensities were not significantly different between the two groups, indicating that basal synaptic function in the amygdala of maternally stressed mice is unaltered $\left(F_{(1,132)}=0.776, p=0.396\right.$ for group differences by RM-ANOVA) (Fig. 6B). We next examined the PPF ratio, an index of presynaptic release probability, using several interpulse intervals $(35,50$, $100 \mathrm{~ms}$, respectively) in the two groups. As shown in Figure 6C, no significant difference in PPF ratio was observed between the two groups $\left(F_{(1,34)}=0.05, p=0.826\right.$ for group differences by RM-ANOVA), suggesting that presynaptic release is normal in maternally stressed mice. Together, these findings suggest that basal synaptic transmission in the thalamo-LA pathway is not significantly changed in maternally stressed mice. 
with the DEX-BSA-treated control mice $\left(F_{(1,627)}=0.002, p=0.97\right.$ for group differences by RM-ANOVA) (Fig. 7C), suggesting a nongenomic action of GC on LTP maintenance.

Based on $3 \mathrm{~h}$ of field recordings in the three treatment groups (vehicle, DEX, and DEX-BSA groups) of maternally stressed and control mice, we summarized the average of the PS amplitude during the first 30 min and last $30 \mathrm{~min}$ after the sixth HFS. As shown in Figure 7D, during the first $30 \mathrm{~min}$, the PS amplitude in all of the tested groups was statistically the same (for controls, $F_{(2,15)}=0.081, p=0.923$; for maternally stressed mice, $F_{(2,17)}=2.99, p=0.077$ by one-way ANOVA). However, post-HFS application of DEX or DEX-BSA restored impaired LTP maintenance in the stressed mice to levels comparable with those seen in the vehicle-treated slices prepared from the control mice (for maternally stressed mice, $F_{(2,17)}=5.77, p<0.05$ by one-way ANOVA) (Fig. 7E), while the same applications did not induce any significant PS amplitude change in the control slices within this LTP protocol, presumably due to the robust synaptic plasticity-evoking condition (for controls, $F_{(2,15)}=0.67, p=$ 0.53 by one-way ANOVA) (Fig. $7 E$ ). Collectively, the restoration of LTP by the post-HFS application of either DEX or DEX-BSA suggests that membraneassociated GRs play a critical role in LTP maintenance in the amygdala of maternally stressed mice.

\section{Nongenomic action of DEX-BSA on auditory fear memory formation}

Given that we had observed the restorative effect of DEX-BSA on LTP in the amygdala of maternally stressed mice, we further examined the effects of DEX-BSA on amygdala-related fear memory formation in both the maternally stressed and control mice. We microinjected DEX-BSA $(0.4 \mu \mathrm{g}$ in $0.2 \mu \mathrm{l})$ or BSA into the BLA through preinstalled cannulas, since DEX-BSA does not penetrate the blood-brain barrier. DEX-BSA was infused immediately after auditory fear conditioning and freezing was monitored $24 \mathrm{~h}$ after conditioning. As shown in Figure 8A, microinjection of DEX-BSA significantly increased the freezing levels in the maternally stressed mice, but not in the control animals $\left(F_{(1,20)}=22.89, p<\right.$ 0.01 for group differences; $F_{(1,20)}=10.68, p<0.01$ for treatment; $F_{(1,20)}=9.565, p<0.01$ for interaction). The freezing levels of the maternally stressed mice that received DEX-BSA were comparable with those seen in the control mice. These results suggest that a nongenomic action of GC restores fear memory consolidation in maternally stressed mice, and that GC signaling cascades, presumably initiated from the amygdaloid membrane, are impaired in these mice.

\section{Discussion}

The present study was designed to elucidate whether maternal stress causes long-lasting alterations of amygdala-dependent emotional learning capabilities in adult rodent offspring. Indeed,
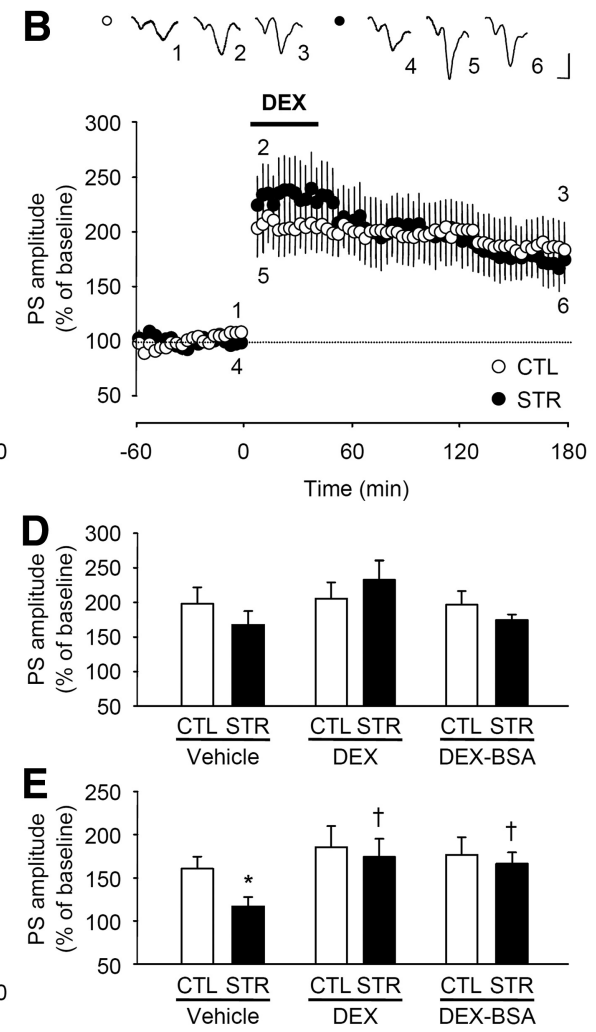

Figure 7. Synaptic plasticity of the thalamo-LA synapses. $A$, HFS-induced PS amplitude ${ }^{*} p<0.05$ vs CTL by Student's $t$ test; Calibration: $2 \mathrm{~ms}, 0.2 \mathrm{mV}$. D, STP expressed as the means \pm SEM of the PS amplitude for the first $30 \mathrm{~min}$ after HFS in each group from the results in $\boldsymbol{A}$ (Vehicle), $\boldsymbol{B}$ (DEX), and $\boldsymbol{C}$ (DEX-BSA). $\boldsymbol{E}$, The means \pm SEM of PS amplitudes for the last $30 \mathrm{~min}$ (from 2.5 to $3 \mathrm{~h}$ ) after HFS application $\left({ }^{*} p<0.05\right.$ vs (TL by Student's $t$ test and ${ }^{\dagger} p<0.05$ vs vehicle-treated STR by Newman-Keuls test).
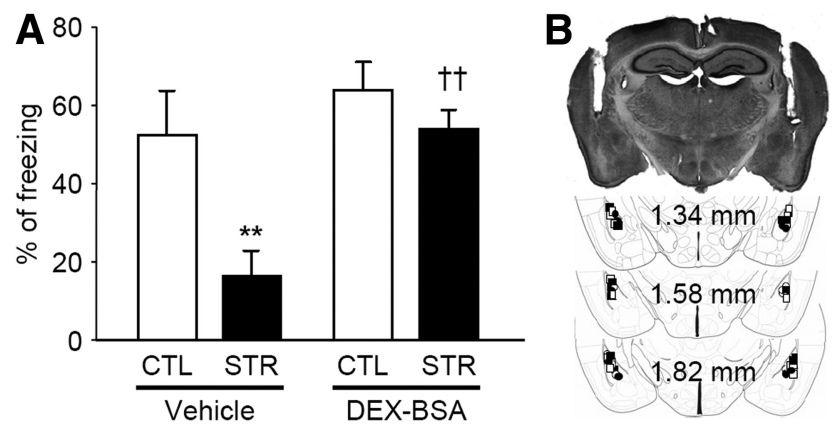

Figure 8. Nongenomic action of $G C$ in auditory fear memory formation as determined using DEX-BSA. $\boldsymbol{A}$, Effects on fear memory formation of microinjection of DEX-BSA immediately after auditory fear conditioning $\left[{ }^{* *} p<0.01\right.$ vs CTL and ${ }^{{ }^{+\dagger}} p<0.01$ vs vehicle-treated STR by Newman-Keuls post hoc comparison; $n=5$ for each vehicle (BSA)-treated group, $n=7$ for each DEX-BSA-treated group]. $\boldsymbol{B}$, A representative photograph and illustrations of the position of an implanted infusion needle. The position is represented by the symbol on three different rostrocaudal planes, with coordinates relative to the mouse bregma $(O$, vehicle-injected control mice; , vehicle-injected maternally stressed mice; $\square$, DEX-BSA-injected control mice; DEX-BSA-injected maternally stressed mice).

male offspring from stressed mothers exhibit impairments in fear memory consolidation and LTP in the thalamo-LA pathway. Interestingly, these abnormalities are rescued by membrane-impermeable DEX-BSA, which is known to activate membrane-initiated GC signaling, suggesting that maternal stress impairs emotional learning, at least in part, by weakening the nongenomic action of GC on the amygdala. Physiologically, the adverse effects of prenatal stress on 
emotional memories, especially fear, suggest that psychiatric disorders related to aversive experiences can be programmed by a stressful environment in utero.

In the auditory fear conditioning paradigm, maternally stressed mice displayed problems in the memory consolidation process, resulting in an LTM defect, in contrast to the normal fear responses during the training period (normal fear memory acquisition) and the retention tests within $2 \mathrm{~h}$ after fear conditioning (STM formation). It is also important to note that the abnormal motor activity and/or innate fear level in maternally stressed mice may compromise the measurement of fear memory, since we used freezing behavior as measure of fear expression. However, it was found in the present study that after habituation, spontaneous motor activity in the testing chamber was not altered within the time period required for testing conditioned fear. It was also demonstrated that prenatal stress does not alter the unlearned fear response to predator odor in adult offspring. Therefore, a neural mechanism responsible for amygdala-dependent memory consolidation is evidently an important substrate of the organizing effects of prenatal stress, although the precise molecular targets require further investigation.

The most striking finding in the present study is the nongenomic effect of GC on the amygdala of maternally stressed mice in the amelioration of fear memory impairment. As shown in Figure 7C, impaired LTP in the thalamo-LA amygdala pathway of stressed offspring was recovered by DEX-BSA treatment. In accord with this finding, bilateral posttraining infusions of DEX-BSA into the amygdala enhanced the conditioned fear response in stressed animals to a level comparable with that of their controls (Fig. 8). These findings provide strong evidence that a membraneinitiated GC signaling in the amygdala, which is attenuated in maternally stressed mice, plays an important role in the regulation of fear memory and synaptic plasticity, independent of GRmediated transcription.

A recent study (Roozendaal et al., 2010) also has suggested a critical role for the nongenomic action of GC on the insular cortex in object recognition learning. In support of these findings, the presence of membrane GR has been demonstrated in an amphibian species (Orchinik et al., 1991). Although membrane GR in mammalian neurons has not been fully characterized, evidence for membrane-associated GR has recently been reported (Riedemann et al., 2010). In this context, it should be noted that Johnson et al. (2005) found GR (classical GR)-immunoreactive sites on the postsynaptic membranes of amygdala neurons. It is therefore plausible that downregulation of putative membrane GR in the amygdala of maternally stressed mice underlies the observed impairments in both fear memory consolidation and LTP. However, whether this putative membrane GR is a membraneassociated form of classical GR, or an as yet unidentified isotype, remains to be determined.

Although the present study is focused on local storage processes in the amygdala, this regional brain tissue, particularly the BLA complex, has been proposed to interact with other brain regions to modulate the memory consolidation related to a variety of emotionally arousing experiences (McGaugh, 2004). For example, Malin and McGaugh (2006) demonstrated an involvement of the amygdala, hippocampus, and anterior cingulate cortex. More recently, critical interactions between the BLA and medial prefrontal cortex (mPFC) were also demonstrated (Roozendaal et al., 2004, 2009). According to this model, amygdala activation during the consolidation phase modulates the activities in other brain regions so as to consolidate acquired memory. Thus, this model may explain why so many different learning tasks are affected by modulation of the amygdala. In fact, in addition to fear-related tasks, object recognition learning has been shown to be affected by modulation of the amygdala (Roozendaal et al., 2008). Therefore, the reduced nongenomic effect of GC on the amygdala of maternally stressed mice may not be limited to the impairment in the consolidation of auditory fear memory investigated here, it may also modulate the consolidation of emotionally influenced memory, possibly by affecting neural activity in other brain regions.

The modulatory effect of GC on memory consolidation appears not to be mediated solely by the amygdala. For example, microinjection of a GR agonist into the MPFC after passive avoidance training rapidly increases ERK1/2 phosphorylation in the BLA within $15 \mathrm{~min}$, and vice versa (Roozendaal et al., 2009). This rapid effect of GR agonists on ERK1/2 phosphorylation, which is also observed in the present study, supports the nongenomic action of GC on memory consolidation processes. Based on these findings, it is tempting to speculate that downregulation of putative membrane GR in the amygdala and perhaps also other interconnected brain regions, is responsible for the impairments in both memory consolidation and conditioning-enhanced ERK1/2 and CREB phosphorylation in maternally stressed mice. Consistently, reduced GR expression in stressed animals was found in various brain regions, including the hippocampus, hypothalamus, and amygdala (see Fig. 5B). Therefore, the reduced expression of GR, possibly membrane-associated GR, in the various interconnected brain regions also may influence neuronal activities reciprocally, thereby dampening the activity of the neural network required for memory consolidation.

In conclusion, the present study clearly demonstrates, for the first time, that prenatal stress produces long-term impairment in both fear memory consolidation and amygdala LTP maintenance in the adult offspring mice. The observed impairments in memory consolidation and LTP appear to involve a reduced nongenomic action of GC on the amygdala of maternally stressed mice. Therefore, our study suggests that the consolidation of emotionally influenced memory is a critical target of the programming effects of maternal stress, resulting in defective behaviors and psychiatric dysfunctions in the offspring.

\section{References}

Bliss TV, Collingridge GL (1993) A synaptic model of memory: long-term potentiation in the hippocampus. Nature 361:31-39.

Chung S, Son GH, Park SH, Park E, Lee KH, Geum D, Kim K (2005) Differential adaptive responses to chronic stress of maternally stressed male mice offspring. Endocrinology 146:3202-3210.

Cratty MS, Ward HE, Johnson EA, Azzaro AJ, Birkle DL (1995) Prenatal stress increases corticotropin-releasing factor (CRF) content and release in rat amygdala minces. Brain Res 675:297-302.

De Kloet ER (2004) Hormones and the stressed brain. Ann N Y Acad Sci 1018:1-15.

de Quervain DJ, Aerni A, Schelling G, Roozendaal B (2009) Glucocorticoids and the regulation of memory in health and disease. Front Neuroendocrinol 30:358-370.

Duvarci S, Paré D (2007) Glucocorticoids enhance the excitability of principal basolateral amygdala neurons. J Neurosci 27:4482-4491.

Fan JM, Chen XQ, Jin H, Du JZ (2009) Gestational hypoxia alone or combined with restraint sensitizes the hypothalamic-pituitary-adrenal axis and induces anxiety-like behavior in adult male rat offspring. Neuroscience 159:1363-1373.

Fanselow MS, LeDoux JE (1999) Why we think plasticity underlying Pavlovian fear conditioning occurs in the basolateral amygdala. Neuron 23:229-232.

Goosens KA, Maren S (2004) NMDA receptors are essential for the acquisition, but not expression, of conditional fear and associative spike firing in the lateral amygdala. Eur J Neurosci 20:537-548.

Huang YY, Martin KC, Kandel ER (2000) Both protein kinase A and mitogen-activated protein kinase are required in the amygdala for the 
macromolecular synthesis-dependent late phase of long-term potentiation. J Neurosci 20:6317-6325.

Joëls M, Baram TZ (2009) The neuro-symphony of stress. Nat Rev Neurosci 10:459-466.

Johnson LR, Farb C, Morrison JH, McEwen BS, LeDoux JE (2005) Localization glucocorticoid receptors at postsynaptic membranes in the lateral amygdala. Neuroscience 136:289-299.

Kim J, Lee S, Park H, Song B, Hong I, Geum D, Shin K, Choi S (2007a) Blockade of amygdala metabotropic glutamate receptor subtype $1 \mathrm{im}-$ pairs fear extinction. Biochem Biophys Res Commun 355:188-193.

Kim J, Lee S, Park K, Hong I, Song B, Son G, Park H, Kim WR, Park E, Choe HK, Kim H, Lee C, Sun W, Kim K, Shin KS, Choi S (2007b) Amygdala depotentiation and fear extinction. Proc Natl Acad Sci U S A 104: 20955-20960.

Kim J, Park S, Lee S, Choi S (2009) Amygdala depotentiation ex vivo requires mitogen-activated protein kinases and protein synthesis. Neuroreport 20:517-520.

LeDoux J (2007) The amygdala. Curr Biol 17:R868-R874.

Malin EL, McGaugh JL (2006) Differential involvement of the hippocampus, anterior cingulate cortex, and basolateral amygdala in memory for context and footshock. Proc Natl Acad Sci U S A 103:1959-1963.

McGaugh JL (2000) Memory—a century of consolidation. Science 287: $248-251$.

McGaugh JL (2004) The amygdala modulates the consolidation of memories of emotionally arousing experiences. Annu Rev Neurosci 27:1-28.

Nathanielsz PW (1999) Life in the womb: the origin of health and disease. Ithaca, NY: Promethean.

Orchinik M, Murray TF, Moore FL (1991) A corticosteroid receptor in neuronal membranes. Science 252:1848-1851.

Pitkänen A, Savander V, LeDoux JE (1997) Organization of intra-amygdaloid circuitries in the rat: an emerging framework for understanding functions of the amygdala. Trends Neurosci 20:517-523.

Riedemann T, Patchev AV, Cho K, Almeida OF (2010) Corticosteroids: way upstream. Mol Brain 3:2.

Rodrigues SM, Schafe GE, LeDoux JE (2004) Molecular mechanisms underlying emotional learning and memory in the lateral amygdala. Neuron 44:75-91.

Rodrigues SM, LeDoux JE, Sapolsky RM (2009) The influence of stress hormones on fear circuitry. Annu Rev Neurosci 32:289-313.

Roozendaal B, Quirarte GL, McGaugh JL (2002) Glucocorticoids interact with the basolateral amygdala beta-adrenoceptor-cAMP/cAMP/PKA system in influencing memory consolidation. Eur J Neurosci 15:553-560.

Roozendaal B, McReynolds JR, McGaugh JL (2004) The basolateral amygdala interacts with the medial prefrontal cortex in regulating glucocorticoid effects on working memory impairment. J Neurosci 24: $1385-1392$.

Roozendaal B, Castello NA, Vedana G, Barsegyan A, McGaugh JL (2008) Noradrenergic activation of the basolateral amygdala modulates consolidation of object recognition memory. Neurobiol Learn Mem 90:576579.

Roozendaal B, McReynolds JR, Van der Zee EA, Lee S, McGaugh JL, McIntyre CK (2009) Glucocorticoid effects on memory consolidation depend on functional interactions between the medial prefrontal cortex and basolateral amygdala. J Neurosci 29:14299-142308.

Roozendaal B, Hernandez A, Cabrera SM, Hagewoud R, Malvaez M, Stefanko DP, Haettig J, Wood MA (2010) Membrane-associated glucocorticoid activity is necessary for modulation of long-term memory via chromatin modification. J Neurosci 30:5037-5046.

Rumpel S, LeDoux J, Zador A, Malinow R (2005) Postsynaptic receptor trafficking underlying a form of associative learning. Science 308:83-88.

Schafe GE, LeDoux JE (2000) Memory consolidation of auditory Pavlovian fear conditioning requires protein synthesis and protein kinase $\mathrm{A}$ in the amygdala. J Neurosci 20:RC96.

Schafe GE, Atkins CM, Swank MW, Bauer EP, Sweatt JD, LeDoux JE (2000) Activation of ERK/MAP kinase in the amygdala is required for memory consolidation of Pavlovian fear conditioning. J Neurosci 20:8177-8187.

Seckl JR (2008) Glucocorticoids, developmental 'programming' and the risk of affective dysfunction. Prog Brain Res 167:17-34.

Son GH, Geum D, Chung S, Kim EJ, Jo JH, Kim CM, Lee KH, Kim H, Choi S, Kim HT, Lee CJ, Kim K (2006) Maternal stress produces learning deficits associated with impairment of NMDA receptor-mediated synaptic plasticity. J Neurosci 26:3309-3318.

Son GH, Chung S, Geum D, Kang SS, Choi WS, Kim K, Choi S (2007) Hyperactivity and alteration of the midbrain dopaminergic system in maternally stressed male mice offspring. Biochem Biophys Res Commun 352:823-829.

Stanciu M, Radulovic J, Spiess J (2001) Phosphorylated cAMP response element binding protein in the mouse brain after fear conditioning: relationship to Fos production. Brain Res Mol Brain Res 94:15-24.

Wallace KJ, Rosen JB (2001) Neurotoxic lesions of the lateral nucleus of the amygdala decrease conditioned fear but not unconditioned fear of a predator odor: comparison with electrolytic lesions. J Neurosci 21:3619-3627.

Weinstock M (2008) The long-term behavioural consequences of prenatal stress. Neurosci Biobehav Rev 32:1073-1086.

Welberg LA, Seckl JR, Holmes MC (2001) Prenatal glucocorticoid programming of brain corticosteroid receptors and corticotrophin-releasing hormone: possible implications for behaviour. Neuroscience 104:71-79. 